\title{
DISTORTION THEOREMS FOR QUASIREGULAR MAPPINGS
}

\author{
RUTH MINIOWITZ
}

\section{Introduction}

In this paper we formulate distortion theorems for quasiregular mappings (qr) in $B^{n}, n \geqq 2$. These theorems hold for every point $x \in B^{n}$ and are of particular interest for points $x$ near the boundary $\partial B^{n}$, unlike distortion theorems of local character; cf. [11].

We first consider non-vanishing qr mappings of bounded degree in Theorem 1 , and prove as a consequence a theorem for non-vanishing and locally quasiconformal mappings in $B^{n}$. This theorem is valid for $n \geqq 3$, but not for $n=2$. Afterwards we present a theorem for qr mappings in $B^{n}$ which omit certain sets, with applications to quasiconformal and qr maps of spherically mean 1-valent.

\section{Notation and terminology}

Notation and terminology are in general as in [5]; in particular, for $x \in R^{n}$ we write $x=\sum_{i=1}^{n} x_{i} e_{i}$, where $e_{1}, \ldots, e_{n}$ are the coordinate unit vectors in $R^{n}$. For $a \in R^{n}$ and $r>0$ we denote $B^{n}(a, r)=\left\{x \in R^{n}:|x-a|<r\right\}, B^{n}(r)=B^{n}(0, r)$, $B^{n}=B^{n}(1), S^{n-1}(a, R)=\partial B^{n}(a, r), S^{n-1}(r)=\partial B^{n}(r)$ and $S^{n-1}=\partial B^{n}$. The closure cl $A$, the boundary $\partial A$ and the complement $\complement A$ of a set $A$ in $R^{n}$ are taken throughout with respect to $R^{n}$. When writing $f: D \rightarrow R^{n}$, we assume throughout that $D$ is a domain in $R^{n}, f$ is continuous and $n \geqq 2$. If $A \subset D, y \in R^{n}$ and $B \subset R^{n}$, we define the following multiplicity (possibly infinite) functions:

$$
\begin{aligned}
N(y, f, A) & =\operatorname{card}\left\{f^{-1}(y) \cap A\right\} \\
N(B, f, A) & =\sup _{y \in B} N(y, f, A) \\
N(f, A) & =N\left(R^{n}, f, A\right) \\
N(f) & =N\left(R^{n}, f, D\right) .
\end{aligned}
$$

The Lebesgue measure of a set $A \subset R^{n}$ will be written as $m_{n}(A)$. 
Let $\Gamma$ be a family of non-constant paths in $R^{n}$. The modulus of $\Gamma$ is defined as

$$
M(\Gamma)=\inf \int_{R^{n}} \varrho^{n} d m_{n}
$$

where the inf is taken over all admissible functions $\varrho$, i.e. non-negative real-valued Borel functions $\varrho$ in $R^{n}$ with

$$
\int_{\gamma} \varrho d s \geqq 1
$$

for all rectifiable $\gamma \in \Gamma$.

For a family of paths lying in a sphere $S=S^{n-1}(x, r)$, the $n$-modulus with respect to $S$ is defined as

$$
M_{n}^{S}(\Gamma)=\inf \int_{S} \varrho^{n} d H^{n-1}
$$

where $H^{n-1}$ is the normalized $(n-1)$-dimensional Hausdorff measure, and the inf is taken over all admissible functions $\varrho$.

$\Gamma(A, B, D)$ denotes the family of all paths which connect $A$ and $B$ in $D$. The modulus of a ring domain, i.e. a domain $R \subset R^{n}$ such that $\complement R$ has exactly two connected components $D_{1}$ and $D_{2}$, is defined as

$$
\bmod R=\left(\frac{\omega_{n-1}}{M\left(\Gamma\left(D_{1}, D_{2}, R\right)\right)}\right)^{1 /(n-1)},
$$

where $\omega_{n-1}=m_{n-1}\left(S^{n-1}\right)$.

\section{Quasiregular mappings}

A mapping $f: D \rightarrow R^{n}$ is said to be quasiregular (qr) if either $f$ is a constant, or else has the following properties:

(i) $f$ is $\mathrm{ACL}^{n}$ (i.e., it is locally absolutely continuous on almost all line segments parallel to the coordinate axes, and its partial derivatives belong to $L_{\mathrm{loc}}^{n}(D)$ ).

(ii) There exists a constant $K \geqq 1$ such that

$$
\left|f^{\prime}(x)\right|^{n} \leqq K J(x, f) \quad \text { a.e. } \quad \text { in } D .
$$

Here $f^{\prime}=\left(\partial f_{i} / \partial x_{j}\right)_{i, j=1}^{n}$ is the formal derivative of $f$, and $\left|f^{\prime}(x)\right|$ denotes the supremum norm of the linear operator $f^{\prime}(x)$ and $J(x, f)=\operatorname{det} f^{\prime}(x)$. A mapping $f: D \rightarrow R^{n}$ is said to be quasiconformal (qc) if $f$ is qr and injective. We denote by $K_{I}(f), K_{o}(f)$ and $K(f)$, respectively, the inner, outer, and maximal dilatation of $f$; see [5]. 


\section{A lower bound for the modulus of the Grötzsch ring domain}

Lemma 1. (Anderson, G. D.) Let $R_{G, n}(r)$ denote the Grötzsch ring whose complementary components are $\complement\left(B^{n}\right)$ and the line segment

Then

$$
E=\left\{x \in B^{n}: 0 \leqq x_{n} \leqq r<1, x_{j}=0,1 \leqq j \leqq n-1\right\} .
$$

$$
D_{n}\{\log [(1+r) /(1-r)]+8 \log 2\}^{1 /(1-n)} \leqq \bmod R_{G, n}(r),
$$

where

$$
D_{n}=\left(\frac{\omega_{n-1}}{\omega_{n-2}}\right)^{1 /(n-1)} \int_{0}^{\pi / 2}(\sin t)^{(2-n) /(n-1)} d t
$$

For $n=2$, the following better estimate holds:

$$
\frac{\pi^{2}}{2}[\log (4(1+r) /(1-r))]^{-1}<\bmod R_{G, 2}(r) .
$$

Proof. From [1, Theorem 2] and [1, Proof to Corollary 1] one can obtain:

$$
\bmod R_{G, n}(r) \geqq D_{n}\left(\frac{1}{\pi^{2}}\right)^{1 /(n-1)}\left(\frac{\pi}{2} \frac{K^{\prime}}{K}\right)^{1 /(n-1)},
$$

where $K$ and $K^{\prime}$ are the complete elliptic integrals

$$
\begin{gathered}
K=K(k)=\int_{0}^{1}\left[\left(1-t^{2}\right)\left(1-k^{2} t^{2}\right)\right]^{-1 / 2} d t, \quad K^{\prime}=K\left(k^{\prime}\right), \\
k=\left(\frac{1-\sqrt{1-r^{2}}}{r}\right)^{1 / 2} \text { and } k^{\prime}=\left(1-k^{2}\right)^{1 / 2} .
\end{gathered}
$$

According to [4, Section 2.1], if we denote

$$
\frac{\pi}{2} \frac{K\left(k^{\prime}\right)}{K(k)}=\mu(k),
$$

then using (2.7) and (2.10) in [4] we obtain:

$$
\mu\left(\left(\frac{\sqrt{1-r^{2}}-(1-r)}{r}\right)^{1 / 2}\right)<\frac{1}{4} \cdot \log \left[\frac{1+r}{1-r} \cdot \frac{2^{7} r^{2}}{(1+r)\left(1-\sqrt{1-r^{2}}\right)}\right] .
$$

It is easy to see that

$$
\frac{1}{2} \leqq \frac{r^{2}}{(1+r)\left(1-\sqrt{1-r^{2}}\right)} \leqq 2
$$

Thus we get:

$$
\bmod R_{G, n}(r) \geqq D_{n}[\log ((1+r) /(1-r))+8 \log 2]^{1 /(1-n)} .
$$


For $n=2$, according to [4, Section 2.2] we have:

$$
\left[\bmod R_{G, 2}(r)\right] \cdot\left[\bmod R_{G, 2}\left(\frac{1-r}{1+r}\right)\right]=\frac{\pi^{2}}{2} ;
$$

therefore, using inequality (2.10) in [4, Section 2.3] we obtain (4.1)'.

The author wishes to thank Prof. G. Anderson for his remarks that yield the final form of Lemma 1.

\section{Distortion theorems for quasiregular mappings of bounded degree and for locally quasiconformal mappings in $B^{n}, n \geqq 3$}

In this section we apply Rickman's method [9] for finding a lower bound for the modulus of a certain family of paths in terms of multiplicity. With that bound and Lemma 1, we shall get a distortion lemma, Lemma 2. An immediate consequence is a distortion theorem, Theorem 1, for qr mappings of bounded degree. Another consequence valid for $n \geqq 3$ but not for $n=2$, is a distortion theorem for locally qc mappings, Theorem 2.

Lemma 2. Let $f: B^{n} \rightarrow R^{n} \backslash\{0\}$ be a K-quasiregular mapping. For $x \in B^{n}$, let

and

$$
A_{x}=\left\{y \in R^{n}:|f(0)| \leqq|y| \leqq|f(x)|\right\} \quad \text { if } \quad|f(0)| \leqq|f(x)|
$$

$$
A_{x}=\left\{y \in R^{n}:|f(x)| \leqq|y| \leqq|f(0)|\right\} \quad \text { if } \quad|f(x)| \leqq|f(0)| .
$$

Also, let $N_{x}=\sup _{y \in A_{x}} N\left(y, f, B^{n}\right)$. Then

$$
\frac{|f(0)|}{A}\left(\frac{1-r}{1+r}\right)^{\beta} \leqq|f(x)| \leqq|f(0)| A\left(\frac{1+r}{1-r}\right)^{\beta}, \quad|x|=r,
$$

where

and

$$
A=2^{8 \beta}
$$

$$
\beta=2^{3 n-1} K_{I}(f) N_{x}^{n+1} .
$$

For $n=2$, the following better estimate holds:

$$
|f(0)| \cdot 2^{-16 K_{I}(f) N_{x}^{3}}\left(\frac{1-r}{1+r}\right)^{32 K_{I}(f) N_{x}^{3}}<|f(x)|<|f(0)| \cdot 2^{16 K_{I}(f) N_{x}^{3}}\left(\frac{1+r}{1-r}\right)^{32 K_{I}(f) N_{x}^{3}} .
$$

Proof. Let $x$ be a point in $B^{n}$ and let $R_{1}=|f(0)|, R_{2}=|f(x)|$ and $E=\{t x: 0 \leqq t \leqq 1\}$. Suppose $R_{2}>R_{1}$; if $R_{1} \geqq R_{2}$, the argument is similar. For $R \in\left[R_{1}, R_{2}\right]$ choose a point $y \in f E \cap S^{n-1}(R)$. If Rickman's path construction [9, Theorem 3.1] is used, there exists a family $\Gamma_{R}$ of paths $\gamma:\left[0, t_{\gamma}\right] \rightarrow S^{n-1}(R)$ such that for any $\varepsilon \in(0,1-|x|)$,

a) $\gamma(0)=y$ if $\gamma \in \Gamma_{R}$,

b) $M_{n}^{S}\left(\Gamma_{R}\right) \geqq \frac{d_{n}}{R N\left(y, f, B^{n}(1-\varepsilon)\right)} n+1$, 
where

$$
d_{n}=\frac{\omega_{n-2}}{2^{3 n-1}}\left(\int_{0}^{\pi / 2}(\sin t)^{-(n-2) /(n-1)} d t\right)^{1-n}
$$

c) Every $\gamma \in \Gamma_{R}$ has a lift $\gamma^{*}$ which starts at $f^{-1}(y) \cap E$ and meets $S^{n-1}(1-\varepsilon)$. Denote $\Gamma_{R}^{*}=\left\{\gamma^{*}: \gamma=f\left(\gamma^{*}\right), \gamma \in \Gamma_{R}\right\}$, and define

$$
\Gamma=\cup\left\{\Gamma_{R}: R_{1} \leqq R \leqq R_{2}\right\}, \quad \Gamma^{*}=\cup\left\{\Gamma_{R}^{*}: R_{1} \leqq R \leqq R_{2}\right\} .
$$

Integration with respect to $R$ yields:

$$
M(\Gamma) \geqq \int_{R_{1}}^{R_{2}} \frac{d_{n}}{N_{x}^{n+1}} \frac{d R}{R}=\frac{d_{n}}{N_{x}^{n+1}} \log \frac{R_{2}}{R_{1}} .
$$

$\Gamma=f\left(\Gamma^{*}\right)$ and $\Gamma^{*} \subset \tilde{\Gamma}=\Gamma\left(E, S^{n-1}(1-\varepsilon), B^{n}(1-\varepsilon)\right) ;$ hence, see [8],

$$
M(\Gamma) \leqq K_{I}(f) M\left(\Gamma^{*}\right) \leqq K_{I}(f) M(\tilde{\Gamma}) .
$$

Since $x \rightarrow x /(1-\varepsilon)$ maps $\mathrm{cl} B^{n}(1-\varepsilon)$ conformally onto $\mathrm{cl} B^{n}$, we have in view of Lemma 1

$$
M(\widetilde{\Gamma}) \leqq\left(D_{n}^{n-1} / \omega_{n-1}\right)^{-1}\left[\log \left\{2^{8}(1-\varepsilon+r) /(1-\varepsilon-r)\right\}\right], \quad r=|x| .
$$

If one lets $\varepsilon \rightarrow 0,(5.2)$ and (5.3) yield:

$$
\left[d_{n} / N_{x}^{n+1}\right] \log \left(R_{2} / R_{1}\right) \leqq K_{I}(f)\left(D_{n}^{n-1} / \omega_{n-1}\right)^{-1}\left[\log \left\{2^{8}(1+r) /(1-r)\right\}\right],
$$

and therefore

$$
|f(x)| \leqq|f(0)| A\left(\frac{1+r}{1-r}\right)^{\beta} .
$$

To find the lower bound, we take $h \circ f$, where $h$ is a composition of two inversions, the first in the sphere $S^{n-1}$ and the second in the plane $x_{n}=0$. The mapping $h \circ f$ is qr with $K_{I}(h \circ f) \leqq K_{I}(f)$, as $h$ is conformal, and $h \circ f: B^{n} \rightarrow R^{n} \backslash\{0\}$ satisfies the conditions of the theorem. Thus

$$
M(r, h \circ f)=\max _{|z| \mp r}|h \circ f(z)| \leqq A|h \circ f(0)|\left(\frac{1+r}{1-r}\right)^{\beta} .
$$

Since $f \neq 0, M(r, h \circ f)=1 / m(r, f)$, where $m(r, f)=\min _{|z|=r}|f(z)|$, and we have the lower bound. For $n=2$ we use the estimate for $n=2$ in Lemma 1 and obtain $(5.1)^{\prime}$. 
Remarks.

(i) Note that $N_{x}$ may be finite for some $x$ 's and infinite for others. The lemma is meaningful for $x$ 's with $N_{x}<\infty$.

(ii) The constants $A$ and $\beta$ in Lemma 2 are probably not the best possible.

An immediate consequence of Lemma 2 is Then

Theorem 1. Let $f: B^{n} \rightarrow R^{n} \backslash\{0\}$ be a $K$-quasiregular mapping with $N=N(f)<\infty$.

where

$$
\frac{|f(0)|}{A}\left(\frac{1-r}{1+r}\right)^{\beta} \leqq|f(x)| \leqq|f(0)| A\left(\frac{1+r}{1-r}\right)^{\beta}, \quad r=|x|,
$$

and

$$
A=2^{8 \beta}
$$

$$
\beta=2^{3 n-1} K_{I}(f) N^{n+1} .
$$

For $n=2$ we can get the estimate $(5.1)^{\prime}$ with $N_{x}=N$.

Remark. For $n=2$ and $K=1$, the theorem reduces to a result on analytic functions which is weaker than the classical result; $\mathrm{cf}$. [3, Theorem 5.1].

Lemma 3. Suppose $n \geqq 3$. Let $f: B^{n} \rightarrow R^{n}$ be a local homeomorphism and $K$-quasiregular and for $r \in(0,1)$ let $N(r)=N\left(f, B^{n}(r)\right)$. Then

$$
N(r)=C(r, n, K) \leqq\left(\frac{\sqrt{n} r}{\psi(n, K)(1-r)}\right)^{n},
$$

where $\psi=\psi(n, K)$ is the universal radius of injectivity for locally $K$-qc mappings in $B^{n}$, and $C(r, n, K)$ is a constant which depends only on $r, n$, and $K$.

Proof. If $n \geqq 3$ and $f: B^{n} \rightarrow R^{n}$ is a $K$-quasiregular local homeomorphism, then $f$ is injective in a ball $B^{n}(\psi(n, K))$, where $\psi=\psi(n, K)$ is a positive number depending only on $n$ and $K$. The existence of $\psi$ is asserted in [7, 2.3] and an estimate is found in [10]. It then follows that for every $x \in B^{n}$ the mapping $f \mid B^{n}(x, \psi \cdot(1-|x|))$ is injective. Hence $N(r)$ is less than the number of cubes with main diagonal $2 \psi \cdot(1-r)$, needed to cover $B^{n}(r)$, and is also less than the given upper bound.

Remark. The upper bound for $N(r)$ presented in Lemma 3 is not the best one. A better estimate, but harder to write out, can be achieved by use of balls of constant hyperbolic radius.

Theorem 2. Suppose $n \geqq 3$. Let $f: B^{n} \rightarrow R^{n} \backslash\{0\}$ be a K-quasiregular local homeomorphism. Then

$$
|f(0)| \cdot 2^{-8 \gamma g(r)}\left(\frac{1-r}{1+3 r}\right)^{\gamma g(r)} \leqq|f(x)| \leqq|f(0)| \cdot 2^{-8 \gamma g(r)}\left(\frac{1+3 r}{1-r}\right)^{\gamma g(r)}, \quad|x|=r
$$


where

$$
\gamma=\frac{2^{3 n-1}(\sqrt{n})^{n(n+1)}}{\psi^{n(n+1)}}
$$

and $\psi=\psi(n, K)$ is the universal radius of injectivity for locally $K$-qc mappings in $B^{n}$,

and

$$
g(r)=\left(\frac{1+r}{1-r}\right)^{n(n+1)}
$$

Proof. From Lemma 3, it follows that for $0<r<1$

$$
N\left(\frac{1+r}{2}\right) \leqq\left(\frac{\sqrt{n}(1+r)}{\psi(1-r)}\right)^{n}
$$

Fix $x \in B^{n}$ with $|x|=r<1$ and let $F: B^{n} \rightarrow R^{n} \backslash\{0\}$ be defined as $F(z)=f(((1+r) / 2) z)$ for $z \in B^{n}$. Then $f(x)=F((2 /(1+r)) x)$ for $|x|<(1+r) / 2$. $F$ satisfies the conditions of Lemma 2 with $N_{x} \leqq C((1+r) / 2, n, K)$. Hence, for $|x|=r$ we have the sought estimate.

Remarks.

(i) Lemma 3 is false for $n=2$, as can be seen from the sequence $e^{k z}, k=1,2, \ldots$.

(ii) Since any function of the form $f(z)=e^{g(z)}$, where $g(z)$ is analytic and $g^{\prime}(z) \neq 0$ for $|z|<1$, satisfies the conditions of Theorem 2 , and since any such function may have an arbitrary growth it follows that Theorem 2 is false for $n=2$. (iii) When this paper was completed S. Rickman pointed out that the following theorem can be proved.

Theorem. Let $f: B^{n} \rightarrow R^{n} \backslash\{0\}$ be $K$-quasiregular and a local homeomorphism. Let $x \in B^{n},|x|=r$ and suppose that $|f(x)|>|f(0)|$. Then

$$
\frac{1}{E(n, K)} \exp \left\{\frac{-1}{(1-r)^{(n+1) /(n-1)}}\right\} \leqq \frac{|f(x)|}{|f(0)|} \leqq E(n, K) \exp \left\{\frac{1}{(1-r)^{(n+1) /(n-1)}}\right\}
$$

where $E(n, K)$ is a positive constant which depends only on $n$ and $K$.

Proof. We let $\alpha_{1}$ and $\alpha_{2}$ be paths as in [9, Remark 4.11], $\alpha_{1}:[0,1] \rightarrow R^{n}$ such that $\alpha_{1}(0)=f(0), \alpha_{1}(1)=0$ and $\alpha_{1}[0,1] \subset \mathrm{cl} B^{n}(|f(0)|), \alpha_{2}:[0,1) \rightarrow R^{n}$ such that $\alpha_{2}(0)=f(x), \alpha_{2}(t) \rightarrow \infty, t \rightarrow 1$, and $\alpha_{2}[0,1) \subset\left\lceil B^{n}(|f(x)|)\right.$. Let $\alpha_{1}^{*}$ and $\alpha_{2}^{*}$ be maximal liftings of $\alpha_{1}$ and $\alpha_{2}$ starting at 0 and $x$, respectively. Let $\Gamma=\Gamma\left(\alpha_{1}^{*}, \alpha_{2}^{*}, B(s) \backslash \operatorname{cl} B(r)\right)$, $s=(1+r) / 2$. Then

$$
M(\Gamma) \geqq c_{n} \log (s / r), \quad \text { see }[11,10.12], \quad M(f \Gamma) \leqq \omega_{n-1} /\{\log [|f(x)| /|f(0)|]\}^{n-1} ;
$$

see $[11,7.5]$. 
Using [5, 3.2] we obtain:

and therefore

$$
M(\Gamma) \leqq K_{o}(f) N(s) M(f \Gamma)
$$

$$
\{\log [|f(x)| /|f(0)|]\}^{n-1} \leqq[A(n, K) N(s)] / \log (s / r) .
$$

Since $\log (s / r) \geqq(1-r) / 4$ when $r$ is near 1 , we obtain by Lemma $3 N(s) \leqq$ $B(n, K) /(1-s)^{-n} ;$ hence

and therefore

$$
\{\log [|f(x)| /|f(0)|]\}^{n-1} \leqq D(n, K) /(1-r)^{n+1},
$$

$$
\frac{|f(x)|}{|f(0)|} \leqq E(n, K) \exp \left\{\frac{1}{(1-r)^{(n+1) /(n-1)}}\right\}
$$

It is also clear that there are in Lemma $2 N_{x}$ for which a better estimate than (5.1) can be obtained by this direct method.

The author wishes to thank Prof. S. Rickman for his remarks.

\section{A distortion theorem for quasiregular mappings which omit certain sets}

Theorem 3. Let $f: B^{n} \rightarrow R^{n} \backslash E$ be a $K$-quasiregular mapping, $E \subset R^{n}$ satisfying $E \cap S^{n-1}(R) \neq \emptyset$ for every $R \geqq 0$. Then

where

$$
\frac{|f(0)|}{C}\left(\frac{1-r}{1+r}\right)^{\alpha} \leqq|f(x)| \leqq|f(0)| C\left(\frac{1+r}{1-r}\right)^{\alpha}, \quad r=|x|,
$$

$$
\alpha=2^{n-1} K_{I}(f) \text { and } C=2^{8 \alpha} .
$$

For $n=2$, the following better estimate holds:

$$
|f(0)| \cdot 4^{-2 k_{I}(f)}\left(\frac{1-r}{1+r}\right)^{2 K_{I}(f)}<|f(x)|<|f(0)| \cdot 4^{2 K_{I}(f)}\left(\frac{1+r}{1-r}\right)^{2 K_{I}(f)}, \quad r=|x| .
$$

Proof. Suppose first that $n \geqq 3$. Fix $x \in B^{n}$ with $|x|=r<1$, and let $x^{*} \in B^{n}$ be such that $|x|=\left|x^{*}\right|$ and that $\left|f\left(x^{*}\right)\right|=M(r, f)=\max _{|z|=r}|f(z)|$. Denote the line segment between the origin and $x^{*}$ by $I$. Then $f(I)$ is a curve in $\mathrm{cl} B^{n}(M(r, f))$ connecting $f\left(x^{*}\right)$ and $f(0)$. Let $A=\operatorname{cl} B^{n}(M(r, f)) \backslash B^{n}(|f(0)|), F=f(I) \cap A$ and $\Gamma^{\prime}=$ $\Gamma(E, F, A)$. Using $[12,10.12]$ we find:

$$
C_{n} \log \{M(r, f) /|f(0)|\} \leqq M\left(\Gamma^{\prime}\right),
$$

where

$$
C_{n}=\omega_{n-2} \cdot 2^{1-n}\left(\int_{0}^{\pi / 2}(\sin t)^{-(n-2) /(n-1)} d t\right)^{1-n} .
$$


$\Gamma^{\prime}$ majorizes the family $\tilde{\Gamma}$ of all paths which connect $F$ and $\partial f B^{n}$ in $A$; hence $M\left(\Gamma^{\prime}\right) \leqq$ $M(\tilde{\Gamma})[12,6.4]$. For each $\tilde{\gamma} \in \tilde{\Gamma}$, there exists a path $\gamma:[0,1) \rightarrow B^{n}$ such that $\gamma(0) \in I$, $\gamma(t) \rightarrow \partial B^{n}$ as $t \rightarrow 1$ and $f(\gamma) \subset \tilde{\gamma}$; see [7, Theorem 3.12]. Let $\Gamma=\{\gamma: \tilde{\gamma} \in \tilde{\Gamma}\}$. Then $f \Gamma \prec \tilde{\Gamma}$, and $[12,6.4]$ we obtain:

$$
M\left(\Gamma^{\prime}\right) \leqq M(\tilde{\Gamma}) \leqq M(f \Gamma) \leqq K_{I}(f) M(\Gamma) .
$$

This, in conjunction with (6.3) and Lemma 1, yields:

$$
C_{n} \log \{M(r, f) /|f(0)|\} \leqq K_{I}(f)\left\{D_{n}^{n-1} / \omega_{n-1}\right\}^{-1}[\log \{(1+r) /(1-r)\}+8 \log 2],
$$

and consequently

where

$$
M(r, f) \leqq|f(0)| \cdot 2^{8 \alpha}\left(\frac{1+r}{1-r}\right)^{\alpha},
$$

Hence

$$
\alpha=2^{n-1} K_{I}(f)
$$

$$
|f(x)| \leqq|f(0)| \cdot 2^{8 \alpha}\left(\frac{1+r}{1-r}\right)^{\alpha} .
$$

We obtain the lower bound from (6.4) as in Lemma 2, by considering $h \circ f$. For $n=2$, we repeat the same construction using (4.1)' instead of (4.1) to estimate $M(\Gamma)$. We find

$$
\frac{2}{\pi} \cdot \log \{M(r, f) /|f(0)|\}<\frac{4}{\pi} \cdot K_{I}(f) \log \{4(1+r) /(1-r)\}
$$

and conclude in the same way as for $n \geqq 3$, and get (6.2).

Remark. For $n=2$ and $K=1$ a similar result was obtained by other methods; cf. [3, Theorem 4.17].

\section{A distortion theorem for non-vanishing qc mappings or spherically mean 1 -valent qr mappings}

Suppose $f: G \rightarrow R^{n}$ is sense-preserving, discrete and open; every $x \in D$ has arbitrarily small normal neighbourhoods $U$ (i.e. domains $U$ with $\operatorname{cl} U \subset G, f \partial U=\partial f U$ and $\left.U \cap f^{-1}(f(x))=\{x\}\right)$ with connected complement in $R^{n}[5,2.9]$.

The local topological index of $f$ at a point $x \in G$, denoted $i(x, f)$, may be defined as

$$
i(x, f)=N(f, U),
$$

where $U$ is any normal neighbourhood for $x$ [5, Theorem 2.12]. Define

$$
n(y, f, G)=\sum_{x \in f^{-1}(y)} i(x, f),
$$


that is, $n(y, f, G)$ counts the number of roots of the equation $f(x)=y$ in $G$ with their multiplicity.

Definition. Let $f: G \rightarrow R^{n}$ be a sense-preserving, discrete and open mapping; $f$ is said to be spherically mean $p$-valent $(p>0)$ if

$$
p(R)=p(R, G, f)=\frac{1}{\omega_{n-1} R^{n-1}} \int_{f G \cap S^{n-1}(R)} n(y, f, G) d \Lambda(y) \leqq p
$$

for every $0<R<\infty$, where $d \Lambda$ is an element of spherical measure of $S^{n-1}(R)$.

For $n=2$ and analytic functions, this definition coincides with circumferentially mean $p$-valent [3, p. 94].

Theorem 4. Let $f: B^{n} \rightarrow R^{n}$ be open, discrete and sense-preserving and spherically mean 1-valent. Then there exists a number $l=l_{f} \in[0, \infty]$ with the following properties:

(i) If $R<l$ and $y \in B^{n}(R)$, then $n\left(y, f, B^{n}\right)=1$.

(ii) If $R \geqq l$, there exists $y_{R} \in S^{n-1}(R)$ such that $n\left(y_{R}, f, B^{n}\right)=0$. If, in addition, $f$ is $q r$, then $l<\infty$.

Proof. Denote $n(y)=n\left(y, f, B^{n}\right)$. We shall show first that the set $\{y: n(y) \geqq M\}$ is open for every finite number $M$. If $n\left(y_{0}\right) \geqq M$, there exists a finite number of points $x_{1}, \ldots, x_{q} \in B^{n}$ such that $f\left(x_{j}\right)=y_{0}, i\left(x_{j}, f\right)=n_{j}, j=1,2, \ldots, q$ and $\sum_{j=1}^{q} n_{j} \geqq M$. Let $U_{j}(r)$ denote the $x_{j}$-component of $f^{-1}\left(B^{n}\left(y_{0}, r\right)\right), j=1,2, \ldots, q$. Then there exists $\delta>0$ such that $U_{j}=U_{j}(\delta), j=1,2, \ldots, q$ are normal neighbourhoods of $x_{1}, \ldots, x_{q}$, respectively. Now for $y \in B^{n}\left(y_{0}, \delta\right)$,

$$
\sum_{x \in f^{-1}(y) \cap U_{j}} i(x, f)=n_{j} \quad \text { and } \quad \sum_{x \in f^{-1}(y)} i(x, f)=\sum_{j=1}^{q} n_{j} \geqq M .
$$

Therefore the set $\{y: n(y) \geqq M\}$ is open.

Suppose $n(a)>1, a \in S^{n-1}(R)$. Then $n(y) \geqq 2$ in some neighbourhood of $a$ relative to $S^{n-1}(R)$. Since $f$ is spherically mean 1 -valent, it follows that $n(y)<1$ for some points $y \in S^{n-1}(R)$. Therefore, if $n(y) \geqq 1$ for all $y \in S^{n-1}(R)$, then $n(y)=1$ for all $y \in S^{n-1}(R)$.

Suppose $n(y)=1$ for all $y \in S^{n-1}(R)$. Then $f$ maps $E=f^{-1}\left(S^{n-1}(R)\right)$ homeomorphically onto $S^{n-1}(R)$. Let $D$ denote the bounded component of $R^{n} \backslash E$. Then $f D=B^{n}(R)$, since $f B^{n} \subset R^{n}$, and $f \partial D=f E=S^{n-1}(R)=\partial f D$. Hence $D$ is a normal domain; so $N(y, f, D) \equiv$ const for $y \in \mathrm{cl} B^{n}(R)$, whereby $n(y)=1$ for every $y \in \mathrm{cl} B^{n}(R)$. Let $I=\left\{R>0: n(y)=1\right.$ for all $\left.y \in S^{n-1}(R)\right\}$; then, in view of the openness of $\{y: n(y) \geqq 1\}$ and the last argument, $I$ is either void or else an open interval. Thus (i) and (ii) hold for $l=\sup I$ when $I \neq \emptyset$, and $l=0$ otherwise.

If $f$ is qr, then $n(y)=1$ for every $y \in B^{n}(l)$, and thus $f$ is qc. It follows that $l<+\infty$, because otherwise the inverse function $f^{-1}$, that is qc, should map $R^{n}$ on $B^{n}$, contradicting Liouville's theorem for qc mappings [6]. 
Remark. If $f$ is analytic and circumferentially mean 1-valent, the theorem reduces to a known result; see [3, Lemma 5.2].

Corollary 1. Let $f: B^{n} \rightarrow R^{n} \backslash\{0\}$ be $K$-quasiconformal or $K$-quasiregular and spherically mean 1-valent. Then

$$
\frac{|f(0)|}{C}\left(\frac{1-r}{1+r}\right)^{\alpha} \leqq|f(x)| \leqq|f(0)| C\left(\frac{1+r}{1-r}\right)^{\alpha} ; \quad r=|x|,
$$

where $\alpha$ and $C$ are the same as in Theorem 3.

For $n=2$, we can get:

$$
|f(0)| \cdot 4^{-2 K_{I}(f)}\left(\frac{1-r}{1+r}\right)^{2 K_{I}(f)}<|f(x)|<|f(0)| \cdot 4^{2 K_{I}(f)}\left(\frac{1+r}{1-r}\right)^{2 K_{I}(f)} .
$$

Proof. The property $f \neq 0$ implies $l_{f}=0$ by Theorem 4; hence all conditions of Theorem 3 are satisfied, and the assertion follows from the latter.

Remarks.

(i) For qc mappings Corollary 1 is better than Theorem 1 with $N=1$.

(ii) Gehring proved (see $[12,18.1]$ ) distortion theorems for qc mappings in general domains in terms of the distortion function $\vartheta_{K}^{n}(r)$. Corollary 1 yields an explicit form of $\vartheta_{K}^{n}(r)$ in certain cases.

(iii) The constants $C$ and $\alpha$ in Corollary 1 are probably not the best possible.

Acknowledgement. This paper is part of a D. Sc. thesis written under the supervision of Professor U. Srebro and submitted to the Senate of the TechnionIsrael Institute of Technology in Mathematics 1978. The author wishes to thank Professor U. Srebro for his help in preparing the paper. The author also wishes to thank Professor S. Rickman and Dr. M. Vuorinen from the University of Helsinki for their useful remarks.

\section{References}

[1] Anderson, G. D.: Extremal rings in $n$-space for fixed and varying $n$. - Ann. Acad. Sci. Fenn. Ser. A I $575,1974,1-21$.

[2] Gehring, F., and J. VÄIIÄLÄ: The coefficients of quasiconformality of domains in space. Acta Math. 114, 1965, 1-70.

[3] Hayman, W. K.: Multivalent functions. - Cambridge University Press, Cambridge, London-New York-Melbourne, 1967.

[4] Lehto, O., and K. I. VirTanen: Quasiconformal mappings in the plane. - Springer-Verlag, Berlin-Heidelberg-New York, 1975.

[5] Martio, O., S. RICKMAN, and J. VäISÄLÄ: Definitions for quasiregular mappings. - Ann. Acad. Sci. Fenn. Ser. A I 448, 1969, 1-40.

[6] MARTIO, O., S. RICKMAN, and J. VÄISÄLÄ: Distortion and singularities of quasiregular mappings. - Ibid. 465, 1970, 1-13. 
[7] Martio, O., S. Rickman, and J. VÄIsÄLÄ: Topological and metric properties of quasiregular mappings. - Ibid. 488, 1971, 1-31.

[8] PoleckIĬ, E. A.: The modulus method for non homeomorphic quasiconformal mappings. Mat. Sb. 83 (125), 1970, 260-270 (Russian).

[9] Rickman, S.: A path lifting construction for discrete open mappings with application to quasimeromorphic mappings. - Duke Math. J. 42 no. 4, 1975, 797-809.

[10] SARvaS, J.: Coefficient of injectivity for quasiregular mappings. - Duke Math. J. 43 no. 1, 1976, 147-158.

[11] SRebro, U.: Quasiregular mappings. - Advances in complex function theory, Lecture Notes in Mathematics 505, Springer-Verlag, Berlin-Heidelberg-New York, 1976, 148163.

[12] VÄISÄL Ä, J.: Lectures on $n$-dimensional quasiconformal mappings. - Lecture Notes in Mathematics 229, Springer-Verlag, Berlin-Heidelberg-New York, 1971.

Technion - Israel Institute of Technology

Department of Mathematics

Haifa

Israel

Received 12 October 1977

Revision received 19 April 1978
Current address:

University of Michigan

Department of Mathematics

Ann Arbor, Michigan 48109

USA 